\title{
Predicción de correlación de variables basado en el análisis dimensional
}

Prediction of variable correlation based on dimensional analysis

Recibido: marzo de 2016 | Revisado: mayo de 2016 | Aceptado: junio de 2016

William F. Villarreal Albitres ${ }^{1}$

1 Universidad de San Martín de Porres - Filial Norte, Chiclayo - Perú wvillarreala@usmp.pe

\begin{abstract}
The objective of this investigation was to demonstrate the use of the Dimensional Analysis as a tool for predicting relevant correlation variables of a phenomenon to conduct experimental research. The friction interface between a rough-grooved surface and bagasse compacted by rollers used in the sugar industry is presented as a case study. Within existing techniques to correlate variables, dimensional analysis was selected to solve problems involving many variables including assumed ones. Fourteen variables were selected for determining the influence on the friction interface. The results based on the Dimensional Analysis showed ten dimensionless relationships. Six variables with strong correlation were found using the heuristic analysis. The correlation found using Dimensional Analysis was validated by the experimental correlation. It is concluded that the prediction of dimensional analysis was reliable and that also applies to cases of solid substances.
\end{abstract}

Keywords: dimensional analysis, bagasse, crushing unit, correlation, interface friction, compaction, Heuristic

\section{Resumen}

El objetivo del trabajo fue demostrar el uso del Análisis Dimensional como herramienta para predecir variables relevantes de correlación de un fenómeno para llevar a cabo una investigación experimental. Se presenta como caso de estudio la interface de fricción entre una superficie rugosa-ranurada y bagazo de caña compactado por rodillos usados en la industria del azúcar. Dentro de las técnicas existentes para correlacionar variables, el Análisis Dimensional fue seleccionado para resolver problemas que involucran muchas variables incluso asumidas. Catorce variables se seleccionaron para determinar la influencia sobre la interface de fricción. Los resultados obtenidos basados en el Análisis Dimensional mostraron diez relaciones adimensionales. Se hallaron seis variables con fuerte correlación usando el análisis heurístico. La correlación hallada por Análisis Dimensional fue validada por correlación experimental. Se concluye que la predicción del Análisis Dimensional fue confiable y que aplica también a casos de sustancias sólidas

Palabras claves: Análisis dimensional, bagazo, unidad de chancado, correlación, interface de fricción, compactación, heurístico 


\section{Introducción}

Las leyes que gobiernan los fenómenos naturales y problemas de ingeniería pueden ser entendidas cuando estos son descritos mediante cantidades físicas: masa, longitud, tiempo, temperatura, corriente eléctrica, fuerza, velocidad, densidad, etc. Revelar una ley física es haber alcanzado una clasificación, correlación y causalidad de interrelación de las cantidades físicas, respectivamente. En consecuencia, una ley física para el entendimiento o solución de determinado problema debe ser expresada en términos matemáticos.

En la vida real, pocos son los problemas de ingeniería que pueden ser resueltos en términos matemático y casi ninguno como fenómenos naturales; por lo que su solución debe alcanzarse usando una combinación de análisis teóricos y datos experimentales y observables. Lo anterior sugiere que toda investigación debe ser planeada y ejecutada de modo descriptivo, correlacional o experimental en su propio laboratorio para interpretar y hacer usos de los datos obtenidos por ellos o de otros, con el fin de que los resultados hallados sean aplicados como sea posible. Uno de los problemas que el investigador o ingeniero afronta cuando planea obtener datos que le permitan tener nuevo conocimiento de las leyes físicas que gobiernan determinado fenómeno, medición y prueba de variables en un proceso; es que desconoce las variables involucradas y su comportamiento en el fenómeno o problema estudiado. Identificar las variables que intervienen en un fenómeno o problema con antelación es evitar el estudio de variables irrelevantes, fallos en los resultados del diseño experimental, gasto oneroso, o alargamiento de los tiempos programados, respectivamente.

Estudiar la correlación de variables de un determinado fenómeno es cuantificar y cualificar el grado de asociación de las variables a fin de predecir su comportamiento y relevancia. Varias técnicas, como herramientas, han sido usadas para la determinación correlativa de variables que intervienen en el estudio de un fenómeno o problema antes de llevar a cabo una investigación experimental: análisis dimensional, análisis de regresión, análisis de varianza, las redes neurales artificiales, y la ingeniería ayudada por computadora, CAE. Todas estas técnicas necesariamente requieren del conocimiento previo de las variables; sin embargo, el análisis dimensional se diferencia del resto por analizar variables que pueden asumirse que intervienen en un fenómeno estudiado ya sean estos relevantes o irrelevantes, respectivamente.

El uso de análisis dimensional como técnica para: (a) establecer una fórmula dimensional para variables físicas, (b) verificar la homogeneidad dimensional de relaciones físicas y ecuaciones en la caracterización de un proceso, y (c) la verificación de unidades de medidas de una variable, está bien documentada (Dobre \& Sánchez, 2007; Gibbins, 2011; Qing-Ming, 2011; Szirtes \& Rózsa, 2006); sin embargo, hasta donde el autor conoce, esta técnica no ha sido usada para aplicarla en un medio sólido-líquido.

El objetivo del presente artículo científico fue demostrar que el Análisis Dimensional es una herramienta para identificar las variables correlativas más relevantes, aplicándolo a un caso de estudio determinación de los factores más relevantes que influían en la interface de fricción entre una superficie ranurada-rugosa y bagazo compactado. En el proceso de fabricación de azúcar, la extracción eficiente de líquido retenido en el bagazo mediante compresiones sucesivas de rodillos está influenciada por la interface de fricción (Kannapiran, 2003; Kent, 2003; Villarreal, 2005).

\section{Materiales y Métodos}

Los siguientes materiales y métodos fueron usados para aplicar el análisis dimensional en la identificación de las variables corre- 
lativas más relevantes del estudio de un fenómeno de desaguado de líquidos analizando la interface de fricción en medios porosos como el bagazo de la cańa de azúcar.

\section{El objeto de estudio}

El objeto de estudio constituyó la última unidad de chancado de caña preparada de una fábrica de azúcar de caña. La Figura 1 muestra una unidad de chancado desaguando líquido del bagazo. El modo de operación de esta unidad, llamada también mollino de caña, es compactar el bagazo entre dos rodillos en disposición triangular a grandes presiones para extraer líquido contenido en el bagazo. Los rodillos metálicos son de superficie ranurada con ángulos agudos girando a bajas revoluciones; mientras que el bagazo es de constitución fibrosa y característica poroso. El interés de los fabricantes de azúcar en desaguar el bagazo (biomasa) con baja humedad (a niveles de 47\% contra del promedio de $51 \%$ ) es usarlo como combustible para generar electricidad. Abundante literatura técnica se ha publicado sobre la influencia tanto operativa como de las características de material sobre el desaguado del bagazo (Hugot, 1986; Kent, 2003); sin embargo, en recientes años se ha publicado que mejorando la interface de fricción entre el bagazo y la geometría del rodillo, se logra un incremento de la reducción de líquido retenido el bagazo (Kannapiran, 2003; Kent, 2003; Kent, 2014).

Los factores que influyeron en estas mejoras no fueron publicados; por lo que fue de interés llevar a cabo una investigación experimental para determinar los agentes relevantes que afectaron el proceso de desaguado.

(a)

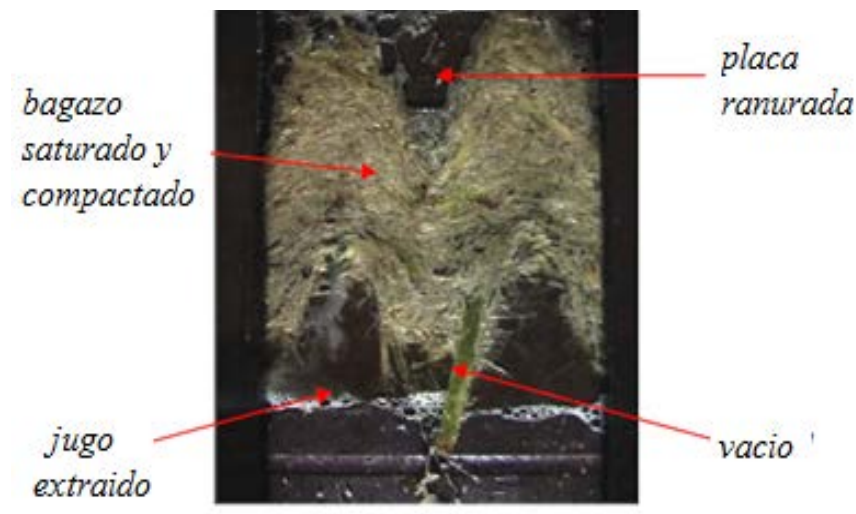

(b)

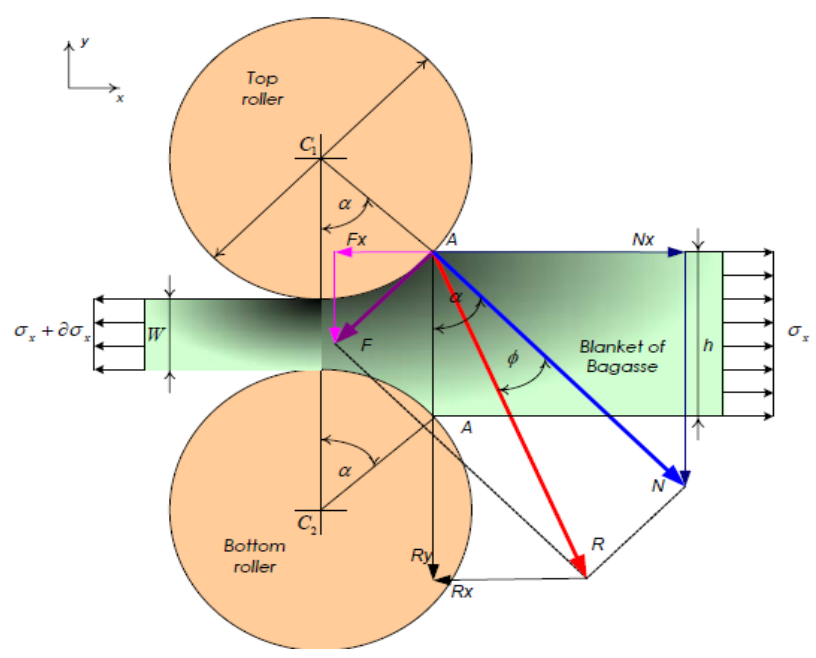

Figura 1. (a) Compactación de bagazo por rodillo ranurado y rugoso mostrando fuerzas de fricción, (b) Vista de desaguado de líquido a través de dientes ranurado (Villarreal, 2003) 


\section{Materiales}

Se usaron los siguientes materiales:

- Una computadora personal de 64bit, 4 GB memoria RAM, 500 DD, 1GB tarjeta gráfica, entorno Windows Seven, versión 2010, Service Pack 1.

- Software comercial MATLAB versión 2013 para evaluar 14 variables por medio matricial

- Datos de resultados experimentales de efecto medición de interface de fricción entre una superficie ranurada y bagazo compactado (Villarreal, 2005)

\section{Métodos y Técnicas}

\section{a. Modelamiento}

El Análisis Dimensional a diferencia de los métodos arriba señalados, no tiene una base teórica, esta se basa en el principio fundamental que cualquier ecuación o relación entre variables es dimensionalmente consistente y similar; es decir, cada término en la relación tiene las mismas dimensiones (Dobre \& Sánchez, 2007). El corolario o consecuencia de este principio es que si toda la ecuación se divide a través de cualquiera de los términos, cada término que queda en la ecuación tiene una relación adimensional.

El análisis dimensional tiene la desventaja de no dar información acerca de la forma de las funciones, ni proporcionar un medio para evaluar constantes de proporcionalidad numérica; sin embargo, el análisis dimensional tiene la ventaja de reducir el número de variables colectándolas en grupos adimensionales. Este es el caso de estudio de problemas de dinámica de fluidos, transferencia de calor o masa, y problemas de mecánica del sólido, en la cual se hace complejo su análisis por las muchas variables que parecen afectar.
La base para la aplicación del análisis dimensional a una gran variedad de problemas se encuentra en el Teorema Pi de Bukingham. El teorema Pi responde a la pregunta de quienes usan el análisis dimensional referente al número de grupos adimensionales que se requieren para reemplazar la lista original de las variables del proceso, y que a letra dice: "si un proceso se caracteriza por una ecuación que involucra $\boldsymbol{m}$ variables físicas, entonces esta ecuación puede reducirse a una relación entre $\boldsymbol{m}$ - $\boldsymbol{n}$ grupos adimensionales independientes, donde $\boldsymbol{n}$ representa el número de dimensiones básicas usadas para describir la variable” (Gibbins, 2011; Qing-Ming, 2011).

Una función definida puede expresar cualquier ley física o principio, si un fenómeno físico tiene $\boldsymbol{n}$ variables independientes $a_{1}, a_{2}, \ldots a_{3}$ y una variable dependiente $V$, entonces $V$ es una función de $a_{1}, a_{2}, \ldots a_{3}$ :

$$
a=f\left(a_{1}, a_{2}, \ldots, a_{k}, a_{k+1}, a_{k+2} \ldots, a_{n}\right)
$$

Si el número de cantidades fundamentales es $\boldsymbol{k}$, es posible sin perder generalidad seleccionar $\boldsymbol{k}$ variables independientes $\boldsymbol{a}_{1}$, $a_{2}, \ldots a_{3}$ con dimensiones independientes entre sí como un grupo de cantidades fundamentales que tienen dimensiones $A_{1}, A_{2}, \ldots$ $A_{\mathrm{k}}$, respectivamente. Las variables independientes restantes $\boldsymbol{n}-\boldsymbol{k}, \boldsymbol{a}_{\mathrm{k}+1}, \boldsymbol{a}_{\mathrm{k}+2}, \ldots, \boldsymbol{a}_{\mathrm{n}}$ son cantidades derivadas con dimensiones:

$$
\begin{array}{ll}
\text { 1. } & a_{k+1}=A_{1}^{p_{1}}, A_{2}^{p_{2}}, \ldots, A_{k}^{p_{k}} \\
\text { 2. } & a_{k+1}=A_{1}^{q_{1}}, A_{2}^{q_{2}}, \ldots, A_{k}^{q_{k}} \\
\text { 3. } & a_{n}=A_{1}^{r_{1}}, A_{2}^{r_{2}}, \ldots, A_{k}^{r_{k}}
\end{array}
$$

donde $p_{1}, \ldots, p_{\mathrm{k}} ; q_{1}, \ldots q_{\mathrm{k}} ; r_{1}, \ldots, r_{\mathrm{k}}=$ valores de potencias relevantes. La variable dependiente $\boldsymbol{a}$ es una cantidad derivada con funciones:

$$
a_{n}=A_{1}^{m_{1}}, A_{2}^{m_{2}}, \ldots, A_{k}^{m_{k}}
$$

donde $m_{1}, \ldots, m_{\mathrm{k}}$ son las potencias relevantes.

Las cantidades fundamentales $a_{1}, a_{2}, \ldots a_{3}$ pueden ser tomadas como un sistema unita- 
rio para medir todas las variables en la ecuación (1): Usando la ecuación (1), las magni- tudes de todas las variables involucradas son números puros adimensionales;

$$
\frac{a}{a_{1}^{m_{1}}, a_{2}^{m_{2}}, \ldots, a_{k}^{m_{k}}}=f\left(1,1, \ldots, 1 ; \frac{a_{k+1}}{a_{1}^{p_{1}}, a_{2}^{p_{2}}, \ldots, a_{k}^{p_{k}}}, \frac{a_{k+2}}{a_{1}^{q_{1}}, a_{2}^{q_{2}}, \ldots, a_{k}^{q_{k}}}, \frac{a_{k+2}}{a_{1}^{r_{1}}, a_{2}^{r_{2}}, \ldots, a_{k}^{r_{k}}}\right)
$$

El término izquierdo de la ecuación (3), $\frac{a_{k+1}}{a_{1}^{p_{1}}, a_{2}^{p_{2}}, \ldots, a_{k}^{p_{k}}}$ es una variable dependiente adimensional denotada como П (pi). En el lado derecho, todas las magnitudes de todas las primeras variables independientes $\boldsymbol{k}$ es igual a 1 y no afecta a $\Pi$. Las magnitudes de las variables independientes $\boldsymbol{n}-\boldsymbol{k}$ restantes, denotadas $\Pi_{1}, \Pi_{2, \ldots} \Pi_{3}$ determina la magnitud de la variable dependiente $\Pi$, donde:

$$
\Pi_{1}=\frac{a_{k+1}}{a_{1}^{p_{1}}, a_{2}^{p_{2}}, \ldots, a_{k}^{p_{k}}} ; \quad \Pi_{2}=\frac{a_{k+1}}{a_{1}^{p_{1}}, a_{2}^{p_{2}}, \ldots, a_{k}^{p_{k}}} ; \Pi_{3}=\frac{a_{k+1}}{a_{1}^{p_{1}}, a_{2}^{p_{2}, \ldots, a_{k}^{p_{k}}}}
$$

Por lo tanto, la variable dependiente adimensional es una función definida de las variable independientes $\boldsymbol{n}-\boldsymbol{k}$ :

$$
\Pi=\Pi_{1}, \Pi_{2}, \ldots, \Pi_{3}
$$

En una función implícita, $f(x, y)=0$, si el numero $N$ de variables $a_{1}, a_{2}, \ldots a_{3}$ relaciona a un problema físico e incluye a una variable dependiente y $\boldsymbol{N}-\mathbf{1}$ variables independientes, la función implícita puede expresar la ley física del problema como:

$$
f\left(a_{1}, a_{2}, \ldots a_{3}\right)=0
$$

en forma reducida:

$$
f\left(1,1, \ldots, 1 ; \Pi_{1}, \Pi_{2}, \ldots, \Pi_{N-k}\right)
$$

la función implícita como función de variables independientes:

$$
f\left(\Pi_{1}, \Pi_{2}, \ldots, \Pi_{N-k}\right)=0
$$

\section{b. Descripción del problema físico y me- todología de resolución}

Un total de 14 variables se identificaron que podrían estar afectando la interface entre el bagazo y la superficie de los rodillos (Figura 1), como se muestra en la Tabla 1.Para validar las variables relevantes halladas por Análisis Dimensional, el siguiente modelo matemático que relaciona el esfuerzo de corte tanto con el coeficiente de fricción, y el esfuerzo normal sobre un plano de deslizamiento (ley de Coulomb), es considerado:

$$
\tau=c+\mu \sigma
$$

donde, $c$ en una constante que representa las propiedades del material y el arreglo de los cuerpos en contacto:

$$
\mu=c+\frac{\tau}{\sigma}
$$


Tabla 1

Lista de variables evaluadas que están presenten en la interface de fricción bagazo y superficie de compactación.

\begin{tabular}{|c|c|c|c|c|}
\hline Variable & Símbolo & Unidad & Dimensión & Observación \\
\hline Coeficiente Fricción & $\mu$ & 1 & 1 & Variab. depent. \\
\hline Esfuerzo de Corte & $\tau$ & $\mathrm{N} / \mathrm{m}^{2}$ & $\mathrm{M}^{0} \mathrm{~F}^{1} \mathrm{~L}^{-2} \mathrm{~T}^{0}$ & Parámetro \\
\hline Ángulo Contacto Inicial & $\alpha_{i}$ & 1 & 1 & Parámetro \\
\hline Altura Penetración Bagazo & $i_{i}$ & $m$ & $\mathrm{M}^{0} \mathrm{~F}^{0} \mathrm{~L}^{1} \mathrm{~T}^{0}$ & Variab. Indep \\
\hline Altura de Aspereza & h & $m$ & $\mathrm{M}^{0} \mathrm{~F}^{0} \mathrm{~L}^{1} \mathrm{~T}^{0}$ & Variab. Indep \\
\hline Ángulo de Ranurado & $\theta$ & 1 & 1 & Variab. Indep \\
\hline Fuerza de Alimentación & F & $N$ & $\mathrm{M}^{0} \mathrm{~F}^{1} \mathrm{~L}^{0} \mathrm{~T}^{0}$ & Parámetro \\
\hline Compactación de bagazo & $\gamma$ & $\mathrm{Kg} / \mathrm{m}^{3}$ & $\mathrm{M}^{1} \mathrm{~F}^{1} \mathrm{~L}^{-3} \mathrm{~T}^{0}$ & Parámetro \\
\hline Esfuerzo Normal & $p$ & $\mathrm{~N} / \mathrm{m}^{2}$ & $\mathrm{M}^{0} \mathrm{~F}^{1} \mathrm{~L}^{-2} \mathrm{~T}^{0}$ & Parámetro \\
\hline Modulo de Corte de Bagazo & $\begin{array}{l}p \\
\mathrm{G}\end{array}$ & $N / m^{2}$ & $\mathrm{M}^{0} \mathrm{~F}^{1} \mathrm{~L}^{-2} \mathrm{~T}^{0}$ & Parámetro \\
\hline Deformación de película de Liquido & $\dot{\rho}$ & $1 / s$ & 1 & Parámetro \\
\hline Velocidad Periférica & & $\mathrm{m} / \mathrm{s}$ & $\mathrm{M}^{0} \mathrm{~F}^{1} \mathrm{~L}^{-2} \mathrm{~T}^{0}$ & Parámetro \\
\hline Densidad de Bagazo & 0 & $\mathrm{~kg} / \mathrm{m}^{3}$ & $\mathrm{M}^{1} \mathrm{~F}^{0} \mathrm{~L}^{-3} \mathrm{~T}^{0}$ & Parámetro \\
\hline Aceleración Gravitacional & G & $\mathrm{m} / \mathrm{s}^{2}$ & $\mathrm{M}^{0} \mathrm{~F}^{0} \mathrm{~L}^{1} \mathrm{~T}^{-2}$ & Parámetro \\
\hline
\end{tabular}

La notación $\mathrm{x}=\mathrm{M}^{\mathrm{a}} \mathrm{F}^{\mathrm{b}} \mathrm{L}^{\mathrm{c}} \mathrm{T}^{\mathrm{d}}$ expresa las dimensiones masa, fuerza, longitud y tiempo

Parámetros son variables que son constantes durante un evento particular.

Aun cuando no es posible definir el modelo matemático que relacione el coeficiente de fricción con las variables independientes, el modelo físico usado para definir la relación fue expresado como función de catorce variables para nuestro caso de estudio:

$$
f(\alpha, \tau, p, \gamma, v, F, H, h, \theta, \rho, \dot{e})
$$

donde $f$ es la función desconocida. La función (7) puede expresarse también como:

$$
f(\alpha, \tau, p, \gamma, v, F, H, h, \theta, \rho, \dot{e})=1
$$

La técnica del análisis dimensional está basada sobre el principio de transformación entre un sistema dimensional (o unidad) y una homogeneidad dimensional (Szirtes \& Rózsa, 2006). Entonces hay un factor numérico para la cantidad dimensional $Q$ en el sistema dimensional 1, y que es equivalente para un sistema dimensional 2. Entonces el problema puede resolverse como

$$
Q \cdot a_{1}^{e_{1}} \cdot a_{2}^{e_{2}}, \ldots, a_{n}^{e}=x . A_{1}^{e_{1}} \cdot A_{2}^{e_{2}}, \ldots, A_{n}^{e_{n}}
$$

donde:

$a_{1} a_{2} \ldots=$ dimensión en sistema 1

$A_{t} A_{2} \ldots=$ dimensión en sistema 2

$e_{1} e_{2} \ldots=$ exponentes de dimensiones en am-

bos sistemas

$n$ : número de dimensiones en cada sistema

$x$ : un factor numérico que expresa equiva-

lente de unidades
Un cambio de sistema de unidades para una variable dimensional $\mathrm{V}_{1}$ puede escribirse como:

$$
V_{1}=a_{1}^{e_{1}} \cdot a_{2}^{e_{1}} \cdot \ldots, a_{n}^{e_{n}}
$$

para un modelo físico $f$ con $\boldsymbol{i}$ variables dimensionales y $n=3$ dimensiones en el sistema:

$$
f\left(V_{1} \cdot V_{2} \cdot, \ldots, V_{i}\right)=f\left(a_{1}^{e_{11}} a_{2}^{e_{21}} a_{3}^{e_{31}} V_{1}, a_{1}^{e_{11}} a_{2}^{e_{21}} a_{3}^{e_{31}} V_{1}, \ldots,\right) a_{1}^{e_{11}} a_{2}^{e_{21}} a_{3}^{e_{31}} V_{1}
$$


Para una relación entre $N_{v}$ variables $V_{1} V_{2}, \ldots$ y $N_{a}$ dimensiones $d_{1} d_{2} \ldots$, la tarea en determinar estas combinaciones particulares (grupos) de variables elevadas a cierta potencia (incógnitas) el cual posee una composición dimensional preseleccionada, es:

$$
\left[V_{1}^{e_{1}} V_{2}^{e_{2}} V_{I}^{e_{n}}\right]=a_{1}^{e_{1}} \cdot a_{2}^{e_{1}} \cdot, \ldots, a_{n}^{e_{n}}
$$

Para este conjunto, catorce variables son determinadas, cuatro dimensiones, y diez productos de variables pueden ser halladas por medio de las ecuaciones simultaneas. Las ecuaciones simultáneas (9) son convertidas a matriz vectorial para hallar las incógnitas $e_{1} e_{2} \ldots e_{n}$ Para calcular las $\Pi$ que darán las variables relevantes de las 14 variables que podrían influir en la interface de fricción. Se requiere procesar

$$
\Pi=f(\alpha, \tau, p, \gamma, v, F, H, h, \theta, \rho, \dot{e})-f(M F L T)
$$

ecuaciones simultaneas; por lo tanto, serán diez valores de según el resultado hallado de la ecuación (10):

$$
\begin{gathered}
k_{11} e_{1}+k_{12} e_{2}+\ldots+k_{1 n} e_{n}=q_{1} \\
k_{21} e_{2}+k_{12} e_{2}+\ldots+k_{2 n} e_{n}=q_{2} \\
\cdot \quad \cdot \quad \cdot \\
\cdot \quad \cdot \\
k_{i 1} e_{1}+k_{i 2} e_{2}+\ldots+k_{i n} e_{n}=q_{n}
\end{gathered}
$$

o como vector

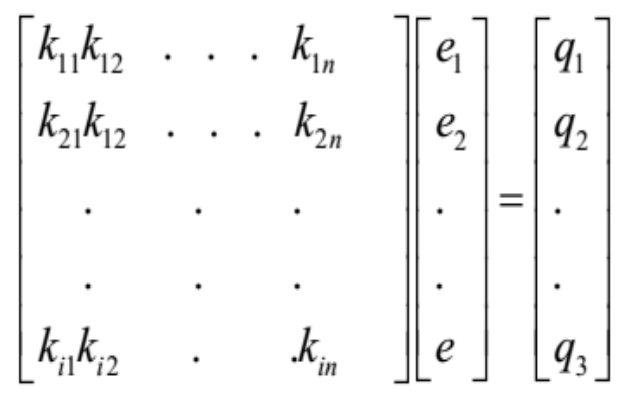

\section{Resultados y Discusión}

\section{Correlación de Variables por Análisis Di- mensional}

La Tabla 2 es el conjunto dimensional o matriz principal que resultó del cálculo matricial por MATLAB de las 14 variables indicadas en la Tabla 1. Esta tabla está dividida en cuatro sub-matrices, de las cuales la sub-matriz inferior izquierda representa el número П’s que nuestro modelo (ecuación 6) tendrá como variables independientes más relevantes. Las otras sub-matrices representaron las consistencia dimensional,

\begin{tabular}{|c|c|c|c|c|c|c|c|c|c|c|c|c|c|c|}
\hline & $\mu$ & $\tau$ & $\alpha_{i}$ & $H$ & $h$ & $\theta$ & $F$ & $\gamma$ & $G$ & $\dot{e}$ & $p$ & $v_{t}$ & $\rho$ & $g$ \\
\hline$M$ & 0 & 0 & 0 & 0 & 0 & 0 & 0 & 1 & 0 & 0 & 0 & 0 & 1 & 0 \\
\hline$F$ & 0 & 1 & 0 & 0 & 0 & 0 & 1 & 0 & 1 & 0 & 1 & 0 & 0 & 0 \\
\hline$L$ & 0 & -2 & 0 & 1 & 1 & 0 & 0 & -3 & -2 & 0 & -2 & 1 & -3 & 1 \\
\hline 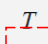 & $0_{-}$ & 0 & 0 & Q & 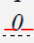 & 0 & - & 0 & $=0$ & 0 & 0 & -1 & 0 & -2 \\
\hline$\pi_{1}$ & 1 & 0 & 0 & 0 & 0 & 0 & 0 & 0 & 0 & 0 & 0 & 0 & 0 & 0 \\
\hline$\pi_{2}$ & 0 & 1 & 0 & 0 & 0 & 0 & 0 & 0 & 0 & 0 & -1 & 0 & 0 & 0 \\
\hline$\pi_{3}$ & 0 & 0 & 1 & 0 & 0 & 0 & 0 & 0 & 0 & 0 & 0 & 0 & 0 & 0 \\
\hline$\pi_{4}$ & 0 & 0 & 0 & 1 & 0 & 0 & 0 & 0 & 0 & 0 & 0 & -2 & 0 & 1 \\
\hline$\pi_{5}$ & 0 & 0 & 0 & 0 & 1 & 0 & 0 & 0 & 0 & 0 & 0 & -2 & 0 & 1 \\
\hline$\pi_{6}$ & 0 & 0 & 0 & 0 & 0 & 1 & 0 & 0 & 0 & 0 & 0 & 0 & 0 & 0 \\
\hline$\pi_{7}$ & 0 & 0 & 0 & 0 & 0 & 0 & 1 & 0 & 0 & 0 & 1 & -4 & 0 & 2 \\
\hline$\pi_{8}$ & 0 & 0 & 0 & 0 & 0 & 0 & 0 & 1 & 0 & 0 & 0 & 0 & -1 & 0 \\
\hline$\pi_{9}$ & 0 & 0 & 0 & 0 & 0 & 0 & 0 & 0 & 1 & 0 & -1 & 0 & 0 & 0 \\
\hline$\pi_{10}$ & $\underline{0}$ & $\underline{0}$ & 0 & $\underline{0}$ & 0 & $\underline{0}$ & 0 & 0 & $\underline{0}$ & 1 & 0 & 1 & 0 & -1 \\
\hline
\end{tabular}
probable no-relevancia y singularidad matricial.

Tabla 2

Matriz dimensional para el coeficiente de fricción para diez variables adimensionales. Las filas son para dimensiones y columnas para variables 
Las variables adimensionales agrupadas

en $\Pi$ son:

$$
\begin{array}{ccccc}
\Pi_{1}=\mu & \Pi_{2}=\frac{\tau}{p} \quad \Pi_{3}=\alpha_{i} & \Pi_{4}=\frac{H g}{v^{2}} & \Pi_{5}=\frac{h g}{v^{2}} \\
\Pi_{6}=0 & \Pi_{7}=\frac{F \dot{e} g^{2}}{p v^{4}} & \Pi_{8}=\frac{\lambda}{\rho} & \Pi_{9}=\frac{G}{p} & \Pi_{10}=\frac{\dot{e} v}{g}
\end{array}
$$

o como función:

$$
\Pi=\Pi_{1} \Pi_{2} \Pi_{3} \Pi_{4} \Pi_{5} \Pi_{6} \Pi_{7} \Pi_{8} \Pi_{9} \Pi_{10} \quad \text { La función } f \text { es una relación algebraica equi- }
$$

donde $f$ es la función aún a ser determinada. valente a:

$$
\Pi=f\left(\mathrm{k}, \Pi_{2}^{n_{2}} \Pi_{3}^{n_{3}} \Pi_{4}^{n_{4}} \Pi_{5}^{n_{5}} \Pi_{6}^{n_{6}} \Pi_{7}^{n_{7}} \Pi_{8}^{n_{8}} \Pi_{9}^{n_{9}} \Pi_{10}^{n_{10}}\right)
$$

donde $k y n_{2}, \ldots, n$ son incógnitas a ser determinadas en la ecuación (11).

Existen tres formas de hallar estas incógnitas: experimentalmente, por análisis y heurísticamente. Puesto que los dos primeros métodos no estuvieron al alcance, se usó el método heurístico, el cual consiste en solucionar problemas de manera informal empleando la experiencia y creatividad. Por ejemplo, es obvio de las relaciones anteriores que los exponentes $n_{2}, n_{3}, n_{5}, n_{6} n_{8}, n_{9} y n_{10}$, deberían estar entre 0 y 1 , de otra manera $\boldsymbol{\mu}$ estaría mal definido. Si la altura $\boldsymbol{b}$ de la aspereza incrementa entonces $\mu$ aumentará a causa de una mayor penetración en el bagazo; de esta forma una mayor intensidad de fuerza requerirá para desplazar la fibra dentro del bagazo. Haciendo el correspondiente razonamiento heurístico a cada variable adimensional y acoplando la ecuación (6) a la ecuación (11) se llega a la siguiente relación:

$$
\Pi=\left(\Pi_{3} \cdot \Pi_{4} \cdot \Pi_{5} \cdot \Pi_{6} \cdot \Pi_{7} \cdot \Pi_{8}\right)+\Pi_{2} \cdot \Pi_{9}
$$

El producto adimensional relaciona el máximo esfuerzo de corte y está expresado como una función tanto del área aparente como real de la superficie ranurada del diente del rodillo como se muestra en la Figura 3. La relación entre el área aparente y real, según la Figura 3 es:

$$
\frac{A_{\text {real }}}{A_{\text {aparente }}}=\frac{n \pi h^{2} \cos \theta / 2}{H}=\mathrm{const} \cdot\left(\frac{\Pi_{5}^{2} \Pi_{2}}{\Pi_{9}}\right)
$$

reemplazando la ecuación (13) en la ecuación (12):

$$
\Pi_{1}=\Pi_{3}+k_{1} \frac{\Pi_{5}^{2} \Pi_{6} \Pi_{10}}{\Pi_{6} \Pi_{8}}+k_{2} \frac{\Pi_{2}}{\Pi_{9}}
$$

por lo tanto, reemplazando los valores adimensionales en la ecuación (14):

$$
\mu=\left[\alpha_{i}+k_{1} \frac{\rho}{\gamma} \cdot \frac{h^{2} \cos \theta / 2}{H \cdot v} \cdot \dot{e}\right]+k_{2}
$$

la ecuación (15) sugiere que el coeficiente de fricción no solo depende de la deformación de corte del material, sino también de la topografía de la superficie del rodillo, el ángulo del acanalado o ranurado de la superficie, la compactación o densificación del material fibroso, la viscosidad del líquido contenido en el bagazo, y la velocidad periférica del rodillo. 


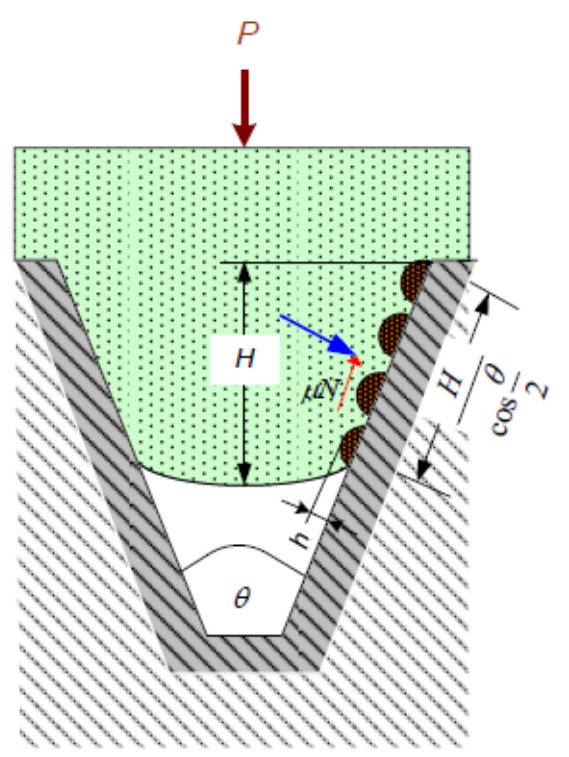

Figura 3. Contacto de área rugosa como función de la penetración del bagazo.

La altura de penetración puede asumirse como una constante, considerando que el material se llena en la ranura de la superficie. Las variables tales como la velocidad periférica, compactación y presión normal causa reducción del coeficiente de fricción, mientras que los otros parámetros lo incrementan. A condiciones iniciales de operación, el coeficiente $\alpha_{l}$, que representa el ángulo de contacto o agarre del material tiene un valor máximo. La compactación $(\gamma)$ parece ser el factor que más influye en el coeficiente de fricción y muestra una tendencia negativa cuando incrementa la compactación del ma- terial. Por lo tanto, la siguiente correlación puede existir para el coeficiente de fricción en un rodillo de molino de cańa:

$$
\mu=f\left(\gamma, \theta, h, p, v, \dot{e} \alpha_{i}\right)
$$

La ecuación 16 expresa que la interface de fricción entre la superficie de los rodillos y el bagazo de cańa existe una fuerte correlación entre la compactación del bagazo, $\theta$, el ángulo, del diente o ranurado de la superficie de los rodillos, la altura, $h$, de dientes del ranurado, la presión hidráulica sobre el bagazo, $p$, que se aplica a los rodillos, la velocidad lineal de giro de los rodillos, ángulo de deformación, , de la película del liquido

\section{Correlación de variables experimentales}

Las Figuras 4 y 5, respectivamente, son los resultados de cinco variables analizadas experimentalmente sobre su efecto sobre la interface de fricción entre la superficie del rodillo y el bagazo compactado. Estos resultados validaron los resultados hallados previamente mediante el Análisis Dimensional, demostrando que este es una técnica que predice confiablemente las variables más relevantes en el estudio de un fenómeno. Respecto a las variables $y$, respectivamente, no se puede predecir su correlación porque no fue incluido en la investigación como variable de estudio.

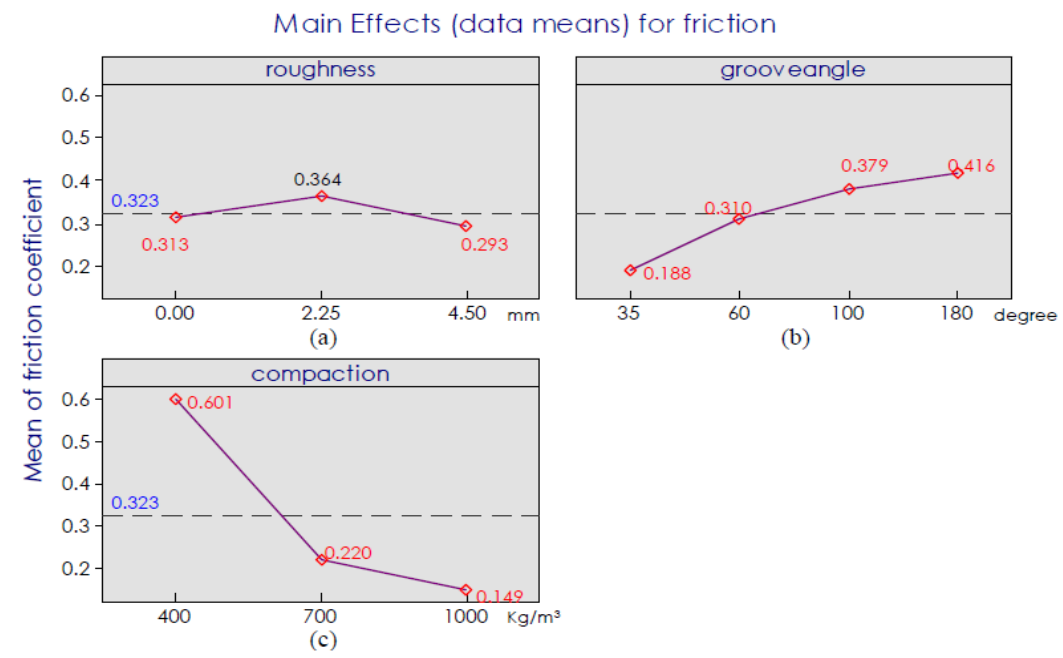

Figura 4. Efecto de la rugosidad de la superficie del rodillo, el ranurado de la superficie y la compactación del bagazo. 
Interaction (data means) for friction coefficient

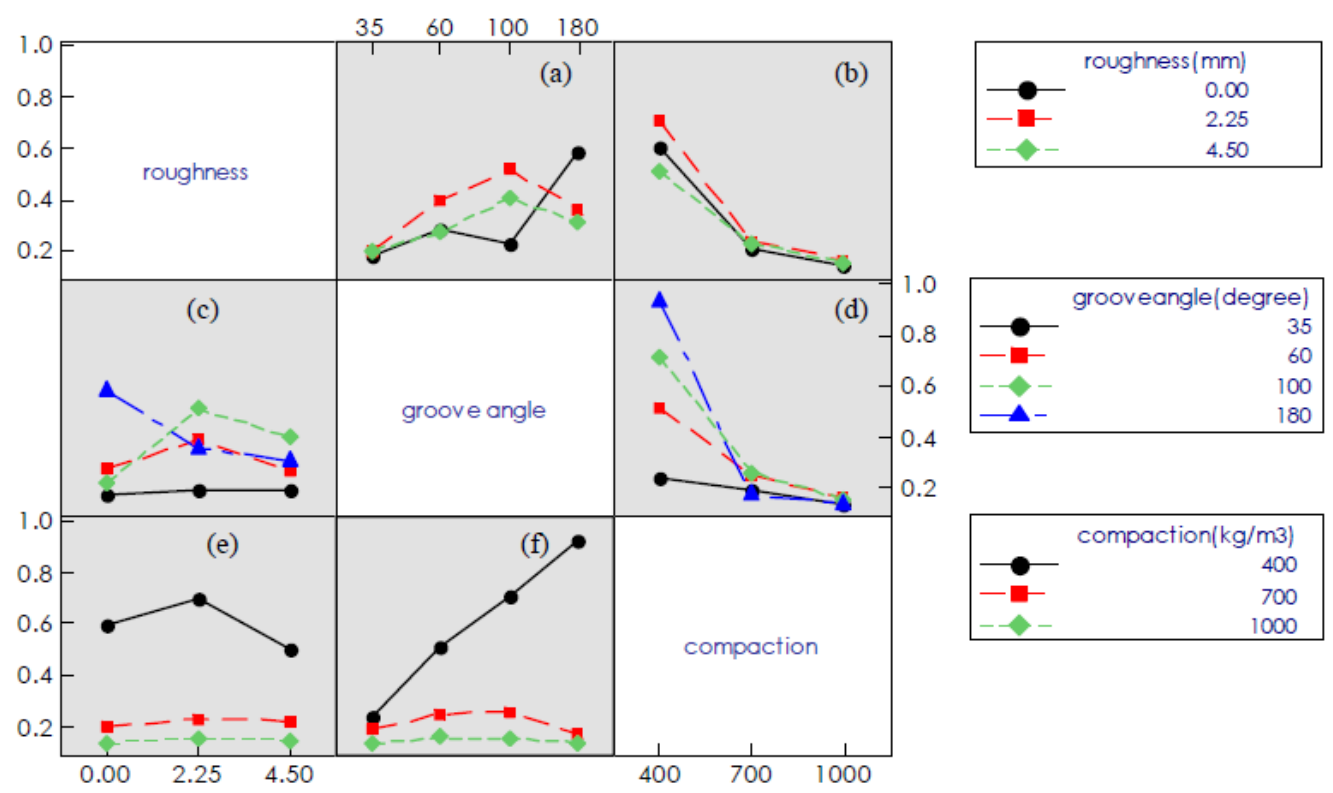

Figura 5. Interacción entre las variables de compactación del bagazo, ranurado de la superficie y rugosidad de la superficie.

\section{Conclusiones}

La técnica del Análisis Dimensional permite predecir con bastante confiabilidad las variables que están correlacionadas en un fenómeno físico.

Los resultados experimentales validaron la correlación existente entre la variable dependiente y las variables independientes predichas por el Análisis Dimensional.
La técnica del análisis dimensional aplica a resolver problemas de la mecánica del sólido.

Los exponentes incógnitas de las variables adimensionales deben ser solucionados heurísticamente.

No se midió el efecto del líquido retenido en el bagazo ni el ángulo de contacto con que agarra el material a ser compactado por los rodillos debido a que no fue parte del proyecto experimental.

\section{Agradecimiento}

El autor desea agradecer al Dr. Jenner Espinoza R., Coordinador Académico de la Escuela de Ingeniería Industrial de la Universidad de San Martín de Porres- Filial Norte, por el apoyo y facilidades brindadas en la publicación de este artículo científico, así como promover la investigación en la Escuela de Ingeniería Industrial. 


\section{Referencias}

Dobre, T. \& Sanchez, J. (2007). Chemical Engineering, Modelling, Simulation, and Similitude. Germany: Wiley-VCH.

Gibbins, J.C. (2011). Dimensional Analysis. U.K.: Springer London.

Hugot, E. (1986). Handbook of Cane Sugar Engineering. Amsterdam: Elsevier Pub.

Kannapiran, A. (2003). Computational and Experimental Modelling of the Crushing of Prepared Sugar Cane (PhD Thesis, James Cook University, Australia).

Kent, G. A. (2003). Increasing the Capacity of Australian raw sugar factory milling sugar (PhD Thesis, James Cook University, Australia).
Kent, G.A. (2014). An alternative Approach to Setting a Mill. Proc S Afr Sug Technol Ass, 87, 172 - 181

Qing-Ming, T. (2011). Dimensional Analysis. Germany: Springer-Verlag.

Szirtes, T. \& Rózsa, P. (2006). Applied Dimensional Analysis and Modeling ( $2^{\mathrm{a}}$ ed.). USA: Elsevier Science \& Technology Books.

Villarreal, W. F. (2005). An experimental investigation into effect of the interface friction on baggase compaction between grooved steel platens (Master Thesis, James Cook University, Australia). 
